\title{
EVALUATION OF EXPOSURE INDEX (lgM) IN ORTHOPAEDIC RADIOGRAPHY
}

\author{
L. Lança ${ }^{1, *}$ and A. Silva ${ }^{2}$ \\ ${ }^{1}$ School of Health Technology, Lisbon Polytechnics, Lisbon, Portugal \\ ${ }^{2}$ Department of Electronic, Telecommunications and Informatics, Aveiro University, Aveiro, Portugal
}

\begin{abstract}
The exposure index (lgM) obtained from a radiographic image may be a useful feedback indicator to the radiographer about the appropriate exposure level in routine clinical practice. This study aims to evaluate lgM in orthopaedic radiography performed in the standard clinical environment. We analysed the $\operatorname{lgM}$ of 267 exposures performed with an AGFA CR system. The mean value of $\operatorname{lgM}$ in our sample is 2.14 . A significant difference $(P=0.000 \leq 0.05)$ from $1.96 \lg M$ reference is shown. Data show that $72 \%$ of exposures are above the $1.96 \mathrm{lgM}$ and $42 \%$ are above the limit of 2.26. Median values of lgM are above 1.96 and below 2.26 for Speed class (SC) 200 (2.16) and SC400 (2.13). The interquartile range is lower in SC400 than in SC200. Data seem to indicate that IgM values are above the manufacturer's reference of 1.96. Departmental exposure charts should be optimised to reduce the dose given to patients.
\end{abstract}

\section{INTRODUCTION}

The purpose of radiation protection is to maintain radiation exposure at the lowest practicable level. The radiation exposure risk should be minimised and this must be guided by two aspects of radiation protection: radiation protection actions and radiation protection principles ${ }^{(1)}$. Radiation protection actions point towards the use of time, shielding and distance to protect patients, personnel and the public. Radiation protection principles deal with the concepts of justification or positive net benefit, optimisation and dose limitation (this concept is applied only for public and occupational exposures).

Exposure optimisation should contribute to protect patients from unnecessary exposures during medical diagnosis and the ALARP (As Low as Reasonably Practicable) principle should always be applied in clinical practice.

For diagnostic purposes, the optimisation of exposure involves the relationship between three core aspects of the imaging process ${ }^{(2)}$ : (i) choice of radiographic technique; (ii) radiation dose to the patient and (iii) diagnostic quality of the radiographic image. These three aspects are determinants of the diagnostic quality of the radiographic image and depend on the radiographer's options for each individual patient examination.

The choice of the most appropriate radiographic technique involves the management of exposure parameters: the patient's radiation exposure and the exposure on the imaging detector to produce the most accurate diagnosis. This means that a correct exposure at the detector should provide optimum image contrast of the radiographic image.

*Corresponding author: luis.lanca@estesl.ipl.pt
In digital imaging systems, the dose delivered to the patient could be an over or underexposure $^{(3)}$ because of their inherent dynamic range. Overexposure might still provide good image quality, but may cause unnecessary dose being delivered to the patient.

Manufacturers provide a wide variation of different exposure index (lgM, log of median exposure) scales to measure the radiation exposure at the detector $^{(4)}$. $\mathrm{LgM}$ is in relation to the absorbed dose at the phosphor plate and is determined by the pixel values $^{(3)}$. For AGFA CR systems, the $\operatorname{lgM}$ provides the dose feedback indicator ${ }^{(5)}$.

\section{Exposure index}

The $\operatorname{lgM}$ value obtained from a radiographic image could be a useful feedback indicator to the radiographer about the appropriate exposure level in routine clinical practice. The AGFA $\lg$ is labelled as $\lg M$ and it indicates how close the actual detector dose is to the expected dose. The $\operatorname{lgM}$ value is related to detector exposure and it does not replace patient's dose-related parameters such as dose-area product (DAP) or entrance skin exposure (ESE). LgM is the logarithm of the median value of the pixel histogram of the segmented image. The analysis of the segmented histogram produces the AGFA dose feedback number that indicates how close the average detector dose in some region of interest behind the patient was to the average detector dose expected from the speed class (SC) used for acquiring that image ${ }^{(5)}$. The relationship between pixel value and exposure must be known: the expected $\operatorname{lgM}$ value for any SC, according to vendor specifications is about 1.96 and should be consistent to a $2.5 \mu \mathrm{Gy}$ exposure measured at the detector ${ }^{(5)}$. Each change of $0.3(\log )$ 


\section{LANÇA AND A. SILVA}

in $\operatorname{lgM}$ corresponds to doubling or halving of dose because of its logarithmic nature. For example, if the $\lg \mathrm{M}$ value for a given image is calculated as 2.26 , it indicates that the dose was about twice that expected for the selected SC. The dose level at the detector is determined as the median of the logarithmic pixel values in the main histogram lobe.

$\mathrm{LgM}$ is related to the $\mathrm{X}$-ray exposure for each radiological projection that is carried out. The patient's exposure must be in order to get a constant dose on the imaging plate (IP) and this varies as a function of patient attributes (e.g. sex, weight) radiographic technique and exposure parameters. LgM value will also vary with the specified SC setting of the digitiser. This means that doubling the exposure dose at the same SC will cause an increase of the $\mathrm{LgM}$ value up to $0.3(\log )$. If the $\mathrm{SC}$ is doubled without modifying the mAs setting, then the $\mathrm{LgM}$ value will decrease by $0.3(\log )^{(6)}$.

Exposures in CR systems may cause unnecessary patient dose due to overexposure. This problem should be avoided in routine clinical practice. If dose is maintained at a relatively constant IP, at a value that is considered to be appropriate for the exam or patient type, then dose consistency in the CR environment could be achieved ${ }^{(5)}$.

The aim of our study was to evaluate $\lg \mathrm{M}$ in orthopaedic radiography performed in a routine clinical environment.

\section{MATERIALS AND METHODS}

In this study, we analysed the $\lg M$ of 267 exposures performed for a period of 1 month. Radiographs were obtained in the routine clinical environment by three experienced radiographers. For statistical reasons, the exposures obtained at an SC of 100 were excluded $(n=2)$ and 265 exposures (125 female and 140 male) obtained at $200 \mathrm{SC}$ and $400 \mathrm{SC}$ were considered. All the radiological projections were acquired using an AGFA CR system (MD-30 image plate).

Exposure information such as patient-related data, examination data, radiographic technique, exposure parameters and post-processing information were recorded in a spreadsheet.

Minitab ${ }^{\circledR} 15$ statistical software was used to perform the statistical analysis. This software provides the Anderson-Darling (AD) test which was used to test the hypotheses that the data $(\operatorname{lgM})$ follow a normal (Gaussian) distribution.

The $\operatorname{lgM}$ reference value used for this study is $1.96^{(5)}$ and a reference standard deviation of $0.2^{(6)}$ was selected for the upper and lower limits for statistical analysis. A significance level of $P \leq 0.05$ was used for statistical tests. Two patient gender groups (female and male) and three patient weight groups (overweight, normal weight and underweight) were considered.

\section{RESULTS}

Table 1 summarises the $\lg \mathrm{M}$ results in orthopaedic radiological examinations considered in this study. The table shows that in the five most requested examinations (knee, foot, pelvis, shoulder and lumbar vertebrae), $\operatorname{lgM}$ mean value is higher than 2.04. As an example, knee is the most requested examination (73) where $\operatorname{lgM}$ mean is higher (2.35) ranging from 1.77 to 2.62 (range 0.85 ). A wide range of $\operatorname{lgM}$ for most of the examinations can be found. There is evidence that the $\lg \mathrm{M}$ range varies considerably among the same examination (e.g. knee, 0.85; pelvis, 0.95; shoulder, 1.11).

The mean value of $\operatorname{lgM}$ in our sample is 2.14 . A sample $t$-test at a significance level of $5 \%$ shows a significant difference $(P=0.000 \leq 0.05)$ from the $1.96 \mathrm{lgM}$ reference value.

Figure 1 shows that median values of $\lg \mathrm{M}$ are above 1.96 and below 2.26 for SC200 (2.16) and SC400 (2.13). The interquartile range is lower in SC400 (0.30) than in SC200 (0.46). The range between upper and lower quartiles is smaller in SC400.

Figure 2 compares $\operatorname{lgM}$ values in two groups of patients (female and male). The $\operatorname{lgM}$ median is highest for females both at 200 (2.36) and 400 SC (2.30). However, in this group of patients, there is no important variability, with an interquartile range of

Table 1. Exposure index.

\begin{tabular}{|c|c|c|c|c|}
\hline Examination & Count & $\begin{array}{c}\text { Mean } \\
(\max -\min )\end{array}$ & SD & Range \\
\hline $\begin{array}{l}\text { Wrist (with } \\
\text { cast) }\end{array}$ & 3 & $2.69(2.76-2.62)$ & 0.10 & 0.14 \\
\hline Leg & 3 & $2.39(2.46-2.33)$ & 0.07 & 0.13 \\
\hline Knee & 73 & $2.35(2.62-1.77)$ & 0.17 & 0.85 \\
\hline Foot & 19 & $2.25(2.59-1.87)$ & 0.21 & 0.72 \\
\hline Elbow & 2 & $2.24(2.26-2.23)$ & 0.02 & 0.03 \\
\hline Wrist & 12 & $2.21(2.53-1.84)$ & 0.25 & 0.69 \\
\hline Pelvis & 17 & $2.13(2.56-1.61)$ & 0.23 & 0.95 \\
\hline Shoulder & 28 & $2.09(2.49-1.38)$ & 0.28 & 1.11 \\
\hline $\begin{array}{l}\text { Pelvis } \\
\text { Orthostatic }\end{array}$ & 6 & $2.07(2.36-1.90)$ & 0.16 & 0.46 \\
\hline Calcaneus & 3 & $2.05(2.30-1.87)$ & 0.22 & 0.43 \\
\hline $\begin{array}{l}\text { Lumbosacral } \\
\text { junction }\end{array}$ & 4 & $2.04(2.30-1.67)$ & 0.31 & 0.63 \\
\hline $\begin{array}{l}\text { Lumbar } \\
\text { vertebrae }^{\mathrm{a}}\end{array}$ & 24 & $2.04(2.62-1.44)$ & 0.29 & 1.18 \\
\hline Ankle & 8 & $2.03(2.43-1.77)$ & 0.20 & 0.66 \\
\hline Hip & 6 & $2.02(2.30-1.51)$ & 0.32 & 0.79 \\
\hline Hand & 4 & $2.00(2.10-1.87)$ & 0.12 & 0.23 \\
\hline $\begin{array}{l}\text { Cervical } \\
\text { vertebrae }\end{array}$ & 14 & $1.93(2.33-1.67)$ & 0.19 & 0.66 \\
\hline Hand digits & 8 & $1.86(2.20-1.38)$ & 0.28 & 0.82 \\
\hline Patella & 25 & $1.86(2.98-1.02)$ & 0.43 & 1.96 \\
\hline Foot digits & 6 & $1.76(1.87-1.61)$ & 0.12 & 0.26 \\
\hline
\end{tabular}

${ }^{\mathrm{a} I n c l u d e s ~ A P}$ and lateral projections. 


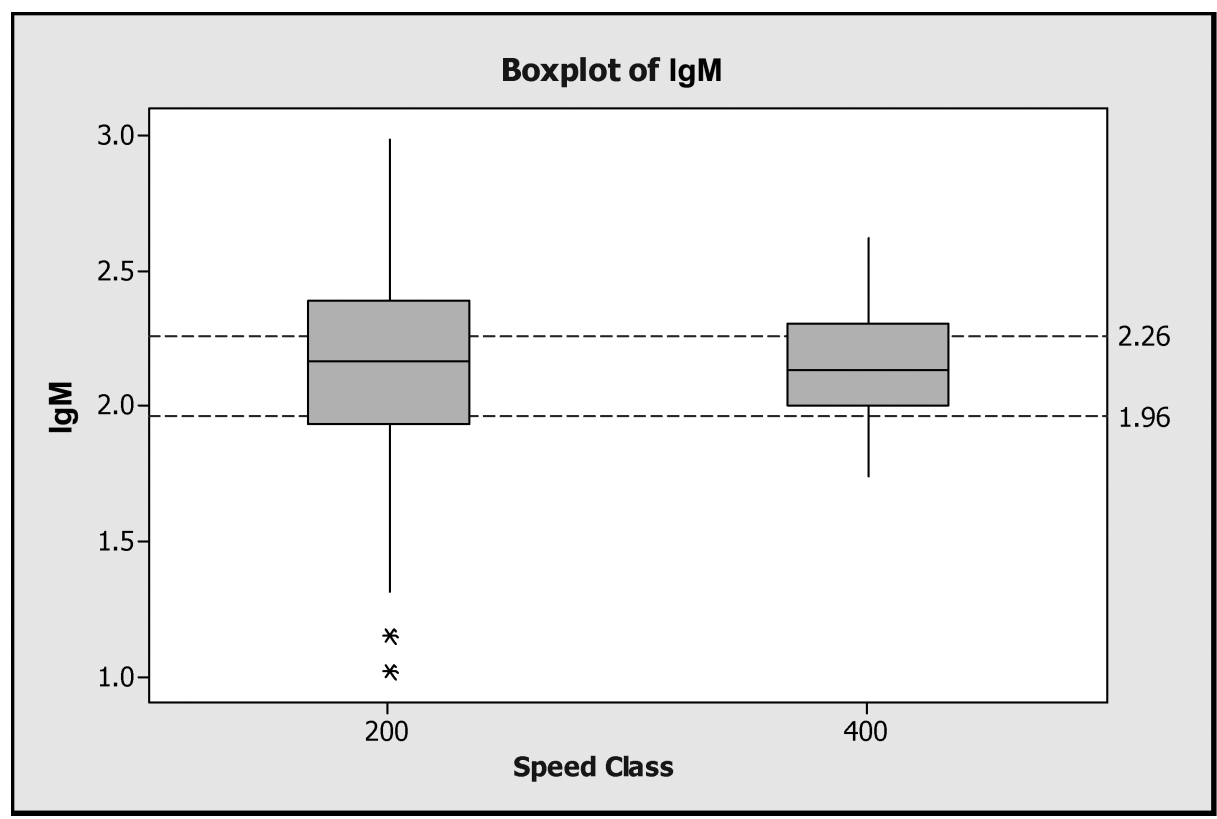

Figure 1. Boxplot of $\operatorname{lgM}$ at 200 and 400 SC. The box contains $50 \%$ of the data. The line inside the box indicates the median value of $\operatorname{lgM}$. The upper edge of the box indicates the 75 th percentile of $\operatorname{lgM}$, and the lower edge indicates the 25 th percentile. The range of the middle two quartiles is the interquartile range. The ends of the vertical lines indicate the minimum and maximum data values. The points outside the ends are outliers.

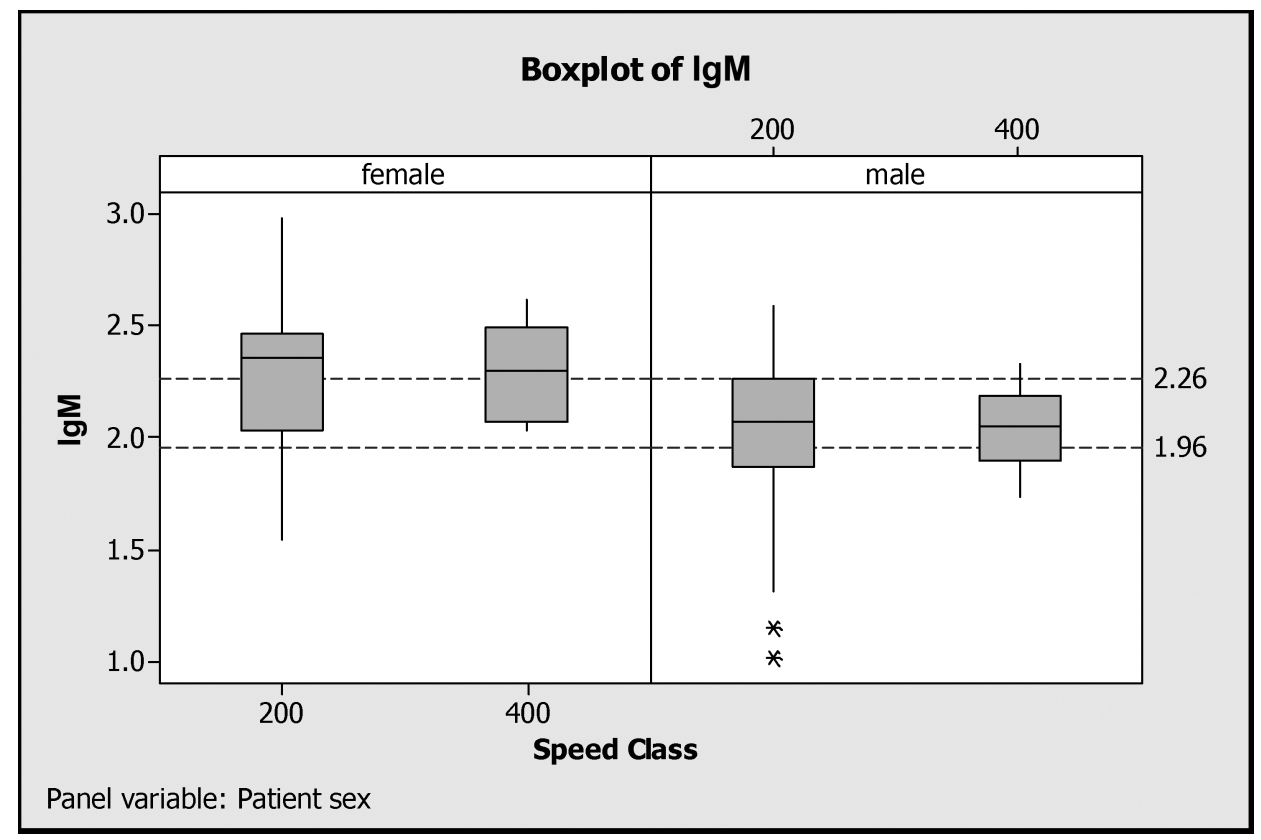

Figure 2. Boxplot of $\operatorname{lgM}$ at 200 and $400 \mathrm{SC}$ comparing female to male patients.

Page 3 of 7 


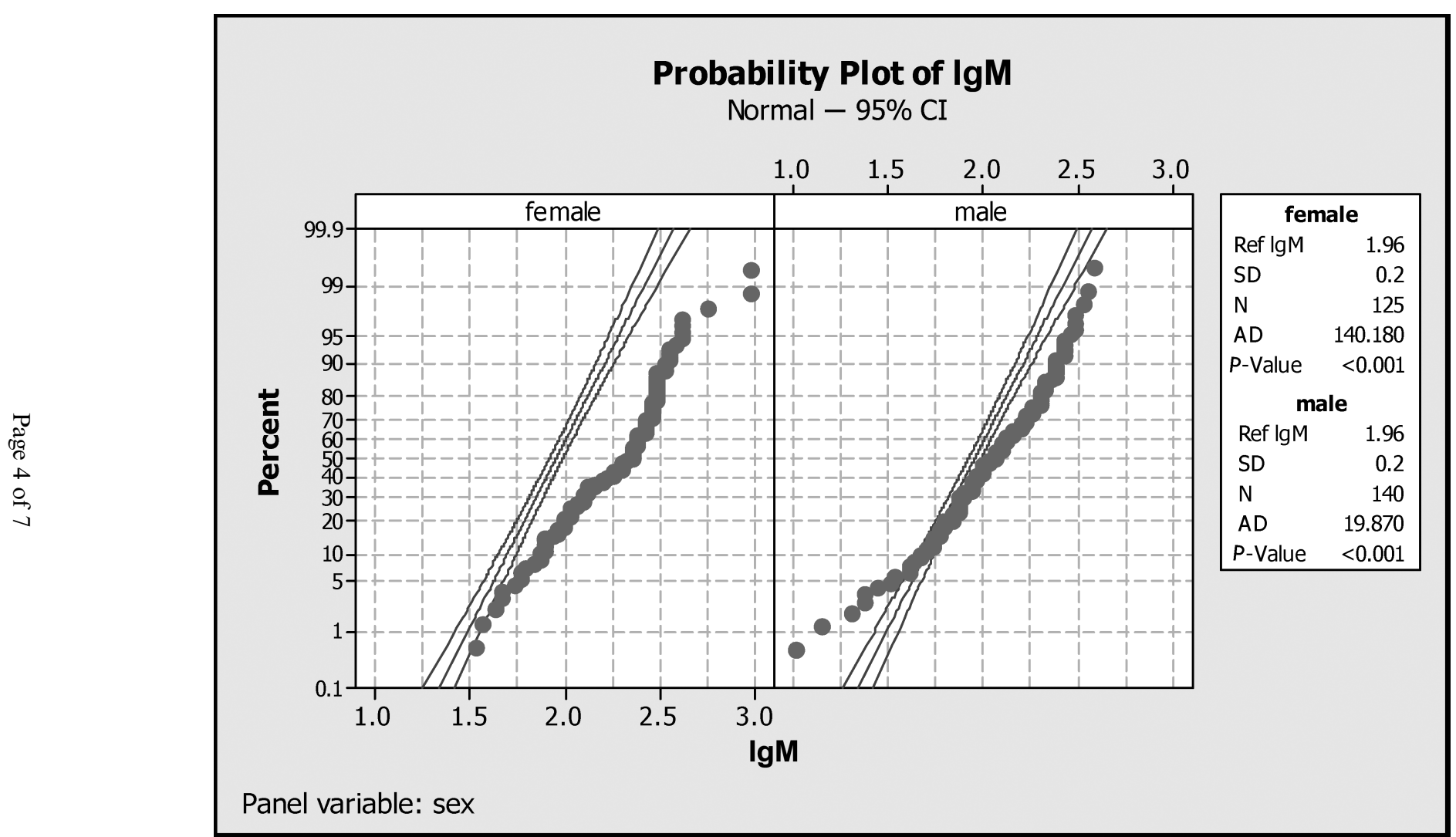

Figure 3. Probability plot of $\operatorname{lgM}$ comparing female to male patients at $1.96 \mathrm{lgM}$ reference. The plot points represent the proportion of lgM failures: note that they do not follow the straight line closely. The fitted line, which is a graphical representation of the percentiles, indicates the $\operatorname{lgM} 1.96$ reference. The lateral lines represent the $95 \%$ confidence intervals (CI). 


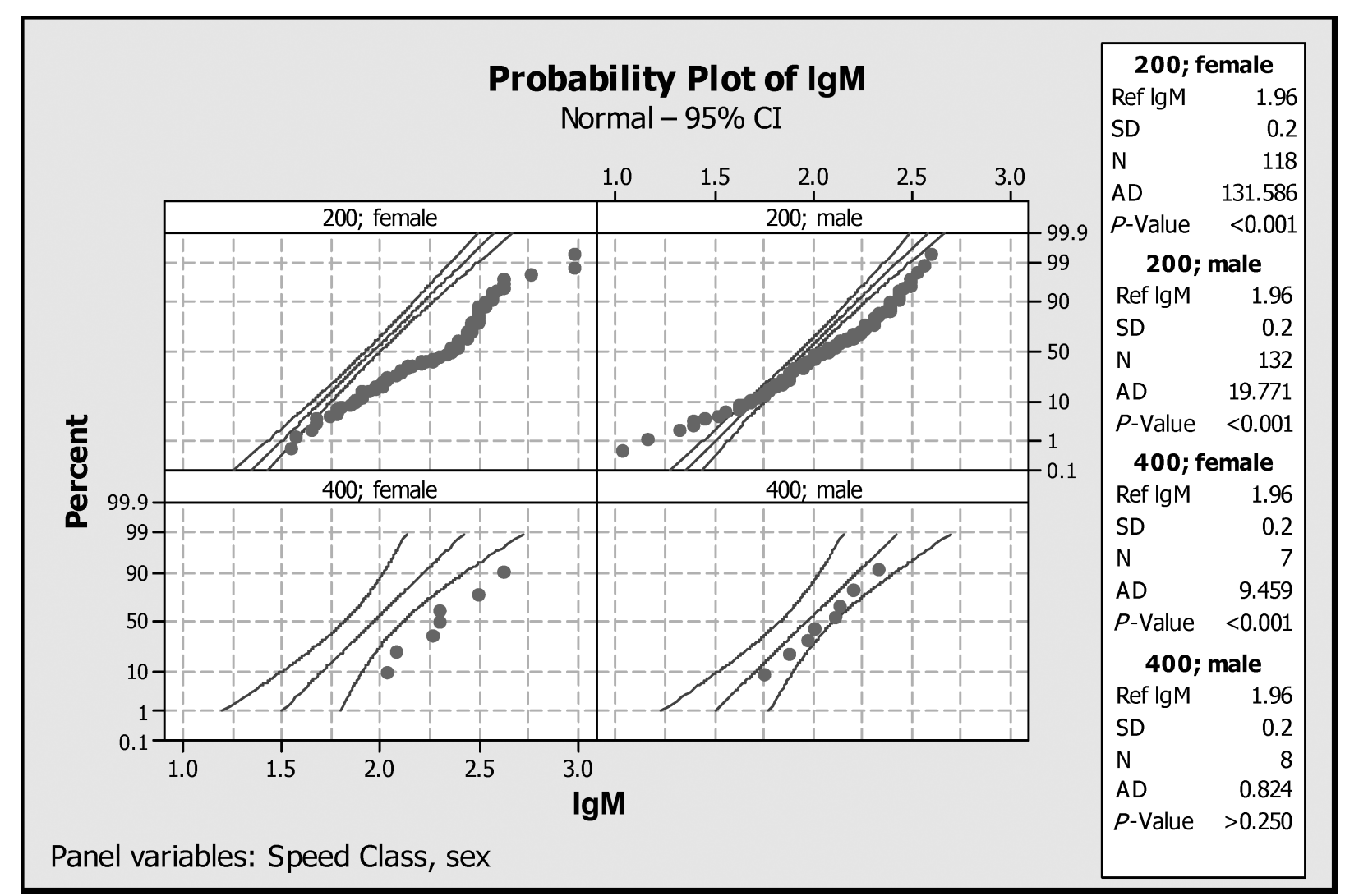

Figure 4. Probability plot of lgM comparing female to male patients at $1.96 \mathrm{lgM}$ reference in SC200 and SC400. 


\section{LANÇA AND A. SILVA}

0.43 (SC200) and 0.42 (SC400). In addition, the $\operatorname{lgM}$ variation between minimum and maximum data values in SC200 (1.54-2.98) is wider than in SC400 (2.03-2.62).

Male patients have similar median $\operatorname{lgM}$ both for SC200 and SC400 (2.07 and 2.05, respectively). SC400 also exhibits the least variability, with an interquartile range of 0.28 and the least difference between minimum and maximum data values (1.742.33). SC200 shows wider interquartile range distribution (0.39) and wider $\operatorname{lgM}$ values for lower and upper boundaries (1.31 and 2.59).

A probability plot of $\operatorname{lgM}$ with the reference value of 1.96 and $0.2 \mathrm{SD}$ is shown in Figures 3 and 4.

If the data fit the normal distribution $\mathrm{AD}$ value will be closer to zero, and the associated $P$-value will be larger than the chosen $P$-level (0.05). The AD statistic test shows that both for female and male patients the data do not follow the specified distribution (Figure 3). Female patients shows an AD value of $140.180(P<0.001)$ and male patients show an AD value of $19.870(P<0.001)$. Despite the fact that results from both groups show a significant difference from the normal distribution, male group of patients had a smaller AD value which indicates that the results from this group are closer to the normal distribution.

Figure 4 shows the $\operatorname{lgM}$ probability plot and compares female with male patients at $1.96 \operatorname{lgM}$ reference in SC200 and SC400, respectively. The results for SC200 show a significant difference $(P<0.001)$ from the normal distribution for both groups of patients. However, lower $\mathrm{AD}$ value is found in male patients (19.771) when compared with female patients (131.586). This means that for SC200 male patient's $\operatorname{lgM}$ is closer to the reference values than the female group.

SC400 shows that the data follow the fitted distribution line fairly closely for male patients. AD statistical value is 0.824 and should be considered as normal $(P>0.250)$. Female patients in SC400 do not fit the reference line. The AD statistical test (9.459) is not normal $(P \leq 0.001)$ showing significant statistical difference from the reference value.

Concerning the patient's weight, a significant statistical difference is found in the normal weight group (female: $n=98 ; \mathrm{AD}=127.496 ; P<0.001$; male: $n=$ 116; $\mathrm{AD}=13.499 ; P<0.001)$. In addition, overweight females $(n=21 ; \mathrm{AD}=20.295 ; P<0.001)$ show a significant difference from 1.96 reference. Although other groups' results do not fit very well with $\operatorname{lgM}$ reference values, no statistically significant differences were found in underweight female $(n=6)$, underweight male $(n=$ 8 ) and overweight male $(n=16)$ groups.

\section{DISCUSSION}

The analysis of the results shows that $\lg \mathrm{M}$ is far above the recommended target of $1.96^{(5)}$. At least
$42 \%$ of evaluated exposures were above the limit of 2.26 , which indicates that the IP receives at least double the exposure necessary to produce an adequate image. Findings also show that $\lg M$ is higher in female patients than in male patients.

This may present a real clinical problem because an $\operatorname{lgM}$ higher than the manufacturer's recommendation could be construed as the routine practice of overexposure. This may be the result of an inadequate exposure chart, particularly for female patients. Exposure parameters and the choice of the most appropriate SC for each examination should be carefully studied in order to obtain the desired image quality at the lowest exposure dose.

The development of an adequate radiographic technique involves the management of exposure parameters, the patient's radiation exposure and the exposure on the imaging detector to produce the most accurate diagnosis. This should be accomplished with an optimisation of exposures and image quality. Further investigations and modifications to exposure charts could lead to a decrease of dose at the detector and a decrease of patient's exposure. Studies performed on a different CR system shows that it is possible to obtain lower exposure indices than those recommended by the manufacturer ${ }^{(7)}$. The establishment of recommended exposure indices remains unclear and the exposure indices used in current clinical practice could be significantly higher than the optimum level.

The $\operatorname{lgM}$ is also sensitive to a number of other factors, the most critical one being segmentation. Any errors in the segmentation algorithm (including background that does not belong to the body part or excluding portions of the body part) can cause variations in $\operatorname{lgM}$. In a related effect, collimation can also affect $\lg \mathrm{M}^{(5)}$. Reliable $\lg \mathrm{M}$ feedback occurs only when the system has been calibrated properly. This study was performed in a routine clinical environment, and data were collected from a CR system that has a normal maintenance programme.

\section{CONCLUSIONS}

Results found in this study seem to indicate that $\lg M$ values are higher than the manufacturer's reference level of 1.96. Departmental exposure charts should be optimised in order to provide a significant reduction of dose at the detector. This action, along with further studies for exposure optimisation should result in a substantial reduction of $\operatorname{lgM}$ and consequently contribute to the reduction of patient radiation exposure.

\section{FUNDING}

This study was supported by AGFA Healthcare Portugal. 


\section{EVALUATION OF EXPOSURE INDEX}

\section{REFERENCES}

1. Seeram, E. and Brennan, P. Diagnostic reference levels in radiology. Radiol. Technol. 77, 373-384 (2006).

2. European Commission (EC). Optimisation of Protection in the Medical Uses of Radiation. EUR 19793 (Luxembourg: Office for Official Publications of the European Communities) (2002).

3. International Commission on Radiological Protection. Managing patient dose in digital radiology. ICRP Publication 93. Ann. ICRP 34(1) (Oxford: Elsevier) (2004).
4. Willis, C. E. Strategies for dose reduction in ordinary radiographic examinations using $C R$ and $D R$. Pediatr. Radiol. 34 (Suppl 3), S196-S200 (2004).

5. Schaetzing, R. Management of paediatric radiation dose using Agfa computed radiography. Pediatr. Radiol. 34 (Suppl 3), S207-S214 (2004).

6. Dose monitoring software user manual (Mortsel, Belgium: Agfa Gevaert) (2003).

7. Peters, S. and Brennan, P. Digital radiography: are the manufacturers' settings too high? Optimisation of the Kodak digital radiography system with aid of the computed radiography dose index. Eur. Radiol. 12, 2381-2387 (2002). 